\title{
Test membranes in Riemann-Cartan spacetimes
}

\author{
Milovan Vasilid* and Marko Vojinovid \\ Institute of Physics, P.O.Box 57, 11001 Belgrade, Serbia
}

(Dated: December 13, 2018)

\begin{abstract}
The dynamics of brane-like extended objects in spacetimes with torsion is derived from the conservation equations of stress-energy and spin tensors. Thus obtained world-sheet equations are applied to macroscopic test membranes made of spinning matter. Specifically, we consider membranes with maximally symmetric distribution of stress-energy and spin. These are characterized by two constants only: the tension and spin magnitude. By solving the world-sheet equations, we discover a similarity between such membranes in Riemann-Cartan backgrounds, and string theory membranes in low-energy string backgrounds. In the second part of the paper, we apply this result to cylindrical membranes wrapped around the extra compact dimension of a $(D+1)$-dimensional spacetime. In the narrow membrane limit, we discover how effective macroscopic strings couple to torsion. An observed similarity with the string sigma model is noted.
\end{abstract}

PACS numbers: 04.40.-b, 11.27.+d

\section{INTRODUCTION}

The problem of motion of brane-like extended objects in backgrounds of nontrivial geometry is addressed by using some form of the Mathisson-Papapetrou method [1, 2]. One starts with the covariant conservation law of the stress-energy and spin tensors of matter fields, and analyzes it under the assumption that matter is localized to resemble a brane. In the lowest, single-pole approximation, the moving matter is viewed as an infinitely thin brane. In the pole-dipole approximation, its non-zero thickness is taken into account.

The known results concerning extended objects in Riemann-Cartan geometry can be summarized as follows. Spinless particles in the single-pole approximation obey the geodesic equation. In the pole-dipole approximation, the rotational angular momentum of the localized matter couples to spacetime curvature, and produces geodesic deviation [1 5]. If particles have spin, the curvature couples to the total angular momentum, and the torsion to the spin alone [6-10]. Higher branes have been considered in 11 13]. In the spinless case, the curvature couples to the internal angular momentum of a thick brane. The coupling disappears in the limit of an infinitely thin brane 11, 12. The dynamics of spinning branes has been investigated in [13]. The spin-torsion coupling has been derived for an arbitrary distribution of spinning matter over the brane.

In this paper, we want to examine infinitely thin branes with maximally symmetric distribution of stress-energy and spin. In the spinless case, such branes are characterized by the tension alone, and are known as the NambuGoto branes [14, 15]. These branes do not couple to torsion, and our idea is to consider their minimal extension characterized by an additional constant - the spin mag-

\footnotetext{
*Electronic address: mvasilic@ipb.ac.rs
}

${ }^{\dagger}$ Electronic address: vmarko@ipb.ac.rs nitude. The analysis along these lines has already been attempted in 13]. The idea has been to try and find the circumstances under which the classical dynamics of strings could possibly resemble the dynamics of [16 21] . Two major results have been obtained. First, the nontrivial string-torsion coupling exists only if the string is made of spinning matter. Second, if the torsion is totally antisymmetric, the form of the obtained dynamics necessarily differs from [16 21].

In this paper, we continue investigating the behaviour of brane-like extended objects in Riemann-Cartan spacetime. We emphasize that our work is not a part of the mainstream string theory considerations. Our brane-like objects are made of conventional matter, and are used to probe Riemann-Cartan geometry. The only connection with string theory is seen in the form of our resulting equations. Namely, we notice a similarity between the motion of macroscopic test membranes in Riemann-Cartan backgrounds and the motion of string theory membranes in the low-energy string backgrounds. Whether this is just a coincidence, or there is more content in this analogy is not the subject of this paper.

In the subsequent paragraphs, we shall investigate the influence of torsion on the dynamics of membrane-like extended objects. Our effort is motivated by the observation that problems encountered in treating strings in Riemann-Cartan spacetime are nicely solved if membranes are considered instead. In particular, the nontrivial projection of the totally antisymmetric spin tensor on the $p$-brane world-sheet is shown to exist only if $p \geq 2$. This form of spin tensor proves to be the basic ingredient in the construction of membranes with maximally symmetric distribution of spin. We apply the $p$-brane equations of [13] to a membrane characterized by two constants only: the tension and spin magnitude. As a result, the behaviour of such membranes in RiemannCartan backgrounds is shown to follow from an action functional. A resemblance with the $\sigma$-model action of [22] is noted. The latter describes a membrane coupled to the string theory 3 -form field. 
The effective string dynamics is obtained in the narrow membrane limit. We consider cylindrical membranes wrapped around the extra compact dimension of a $(D+1)$-dimensional spacetime, and perform a $D+1 \rightarrow D$ dimensional reduction. As a result, a $\sigma$-model action similar to that of [16 21] is revealed.

The layout of the paper is as follows. In section [II, we review the basic notions of the multipole formalism developed in [11 13]. The manifestly covariant worldsheet equations and boundary conditions are explicitly displayed. In section III the $p=2$ brane is investigated. The free coefficients of the theory are chosen to define a Nambu-Goto membrane with totally antisymmetric spin tensor parallel to the world-sheet. This membrane is characterized by the constant tension and spin, and its dynamics in Riemann-Cartan geometry is shown to resemble the dynamics of elementary membranes in the low-energy string backgrounds [22]. In section IV] a narrow membrane wrapped around the extra compact dimension of a $(D+1)$-dimensional spacetime is considered. An effective string dynamics is revealed in the limit of the small extra dimension. Section $\nabla$ is devoted to concluding remarks.

Our conventions are the same as in [12]. Greek indices $\mu, \nu, \ldots$ are the spacetime indices, and run over $0,1, \ldots, D-1$. Latin indices $a, b, \ldots$ are the worldsheet indices and run over $0,1, \ldots, p$. Latin indices $i, j, \ldots$ refer to the world-sheet boundary and take values $0,1, \ldots, p-1$. The coordinates of spacetime, world-sheet and world-sheet boundary are denoted $x^{\mu}, \xi^{a}$ and $\lambda^{i}$ respectively. The spacetime metric is denoted by $g_{\mu \nu}(x)$, and the induced world-sheet metric by $\gamma_{a b}(\xi)$. The signature convention is defined by $\operatorname{diag}(-,+, \ldots,+)$, and the indices are raised using the inverse metrics $g^{\mu \nu}$ and $\gamma^{a b}$.

\section{MULTIPOLE FORMALISM}

It has been shown in 11, 12] that an exponentially decreasing function can be expanded as a series of $\delta$ function derivatives. For example, a tensor-valued function $F^{\mu \nu}(x)$, well localized around the $p+1$-dimensional surface $\mathcal{M}$ in $D$-dimensional spacetime, can be decomposed in a manifestly covariant way as

$$
\begin{aligned}
F^{\mu \nu}(x)= & \int_{\mathcal{M}} d^{p+1} \xi \sqrt{-\gamma}\left[M^{\mu \nu} \frac{\delta^{(D)}(x-z)}{\sqrt{-g}}-\right. \\
& \left.-\nabla_{\rho}\left(M^{\mu \nu \rho} \frac{\delta^{(D)}(x-z)}{\sqrt{-g}}\right)+\ldots\right] .
\end{aligned}
$$

The surface $\mathcal{M}$ is defined by the equation $x^{\mu}=z^{\mu}(\xi)$, where $\xi^{a}$ are the surface coordinates, and the coefficients $M^{\mu \nu}(\xi), M^{\mu \nu \rho}(\xi), \ldots$ are spacetime tensors called multipole coefficients. Here, and throughout the paper, we make use of the surface coordinate vectors

$$
u_{a}^{\mu} \equiv \frac{\partial z^{\mu}}{\partial \xi^{a}},
$$

and the surface induced metric tensor

$$
\gamma_{a b}=g_{\mu \nu} u_{a}^{\mu} u_{b}^{\nu}
$$

The induced metric is assumed to be nondegenerate, $\gamma \equiv \operatorname{det}\left(\gamma_{a b}\right) \neq 0$, and of Minkowski signature. The same holds for the target space metric $g_{\mu \nu}(x)$ and its determinant $g(x)$. The covariant derivative $\nabla_{\rho}$ is defined by the Levi-Civita connection.

It has been shown in [12] that one may truncate the series in a covariant way in order to approximate the description of matter. Truncation after the leading term is called single-pole approximation, truncation after the second term is called pole-dipole approximation. In the single-pole approximation, one assumes that the brane has no thickness, which means that matter is localized on a surface. All higher approximations, including poledipole, allow for a nonzero thickness, and thus, for the transverse internal motion.

The decomposition (11) is particularly useful for the description of brane-like objects in spacetimes of general geometry. In [13], the pole-dipole approximation has been used to model the fundamental matter currents - stressenergy tensor $\tau^{\mu}{ }_{\nu}$, and spin tensor $\sigma^{\lambda}{ }_{\mu \nu}$. The brane dynamics in Riemann-Cartan backgrounds is obtained from the covariant conservation laws

$$
\begin{gathered}
\left(D_{\nu}+\mathcal{T}^{\lambda}{ }_{\nu \lambda}\right) \tau^{\nu}{ }_{\mu}=\tau^{\nu}{ }_{\rho} \mathcal{T}^{\rho}{ }_{\mu \nu}+\frac{1}{2} \sigma^{\nu \rho \sigma} \mathcal{R}_{\rho \sigma \mu \nu} \\
\left(D_{\nu}+\mathcal{T}^{\lambda}{ }_{\nu \lambda}\right) \sigma^{\nu}{ }_{\rho \sigma}=\tau_{\rho \sigma}-\tau_{\sigma \rho} .
\end{gathered}
$$

Here, $D_{\nu}$ is the covariant derivative with the nonsymmetric connection $\Gamma^{\lambda}{ }_{\mu \nu}$, which acts on a vector $v^{\mu}$ according to the rule $D_{\nu} v^{\mu} \equiv \partial_{\nu} v^{\mu}+\Gamma^{\mu}{ }_{\lambda \nu} v^{\lambda}$. The torsion $\mathcal{T}^{\lambda}{ }_{\mu \nu}$ and curvature $\mathcal{R}^{\mu}{ }_{\nu \rho \sigma}$ are defined in the standard way:

$$
\begin{aligned}
& \mathcal{T}^{\lambda}{ }_{\mu \nu} \equiv \Gamma^{\lambda}{ }_{\nu \mu}-\Gamma^{\lambda}{ }_{\mu \nu}, \\
& \mathcal{R}^{\mu}{ }_{\nu \rho \sigma} \equiv \partial_{\rho} \Gamma^{\mu}{ }_{\nu \sigma}-\partial_{\sigma} \Gamma^{\mu}{ }_{\nu \rho}+\Gamma^{\mu}{ }_{\lambda \rho} \Gamma^{\lambda}{ }_{\nu \sigma}-\Gamma^{\mu}{ }_{\lambda \sigma} \Gamma^{\lambda}{ }_{\nu \rho} .
\end{aligned}
$$

The covariant derivative $D_{\nu}$ is assumed to satisfy the metricity condition $D_{\lambda} g_{\mu \nu}=0$. As a consequence, the connection $\Gamma^{\lambda}{ }_{\mu \nu}$ is split into the Levi-Civita connection $\left\{\begin{array}{c}\lambda \\ \mu \nu\end{array}\right\}$ and the contorsion $K^{\lambda}{ }_{\mu \nu}$ :

$$
\begin{aligned}
\Gamma^{\lambda}{ }_{\mu \nu} & =\left\{\begin{array}{c}
\lambda \\
{ }_{\mu \nu}
\end{array}\right\}+K^{\lambda}{ }_{\mu \nu}, \\
K^{\lambda}{ }_{\mu \nu} & \equiv-\frac{1}{2}\left(\mathcal{T}^{\lambda}{ }_{\mu \nu}-\mathcal{T}_{\nu}{ }_{\mu}{ }+\mathcal{T}_{\mu \nu}{ }^{\lambda}\right) .
\end{aligned}
$$

The curvature tensor can then be rewritten in terms of the Riemann curvature $R^{\mu}{ }_{\nu \rho \sigma} \equiv \mathcal{R}^{\mu}{ }_{\nu \rho \sigma}(\Gamma \rightarrow\{\})$, and the Riemannian covariant derivative $\nabla_{\mu} \equiv D_{\mu}(\Gamma \rightarrow\{\})$ :

$$
\mathcal{R}^{\mu}{ }_{\nu \lambda \rho}=R^{\mu}{ }_{\nu \lambda \rho}+2 \nabla_{[\lambda} K^{\mu}{ }_{\nu \rho]}+2 K^{\mu}{ }_{\sigma[\lambda} K^{\sigma}{ }_{\nu \rho]} .
$$

Given the system of conservation equations (2), one finds that the second one has no dynamical content. Indeed, the antisymmetric part of the stress-energy tensor is completely determined by the spin tensor. One can 
use (2b) to eliminate $\tau^{[\mu \nu]}$ from (2a), and thus obtain the conservation equations in which only $\tau^{(\mu \nu)}$ and $\sigma^{\lambda \mu \nu}$ appear. The resulting equation has the form

$$
\begin{gathered}
\nabla_{\nu}\left(\theta^{\mu \nu}-K_{\lambda \rho}^{[\mu} \sigma^{\rho \lambda \nu]}-\frac{1}{2} K_{\lambda \rho}{ }^{[\mu} \sigma^{\nu] \rho \lambda}\right)= \\
=\frac{1}{2} \sigma_{\nu \rho \lambda} \nabla^{\mu} K^{\rho \lambda \nu}
\end{gathered}
$$

where $\theta^{\mu \nu}=\theta^{\nu \mu}$ stands for the generalized Belinfante tensor:

$$
\theta^{\mu \nu} \equiv \tau^{(\mu \nu)}-\nabla_{\rho} \sigma^{(\mu \nu) \rho}-\frac{1}{2} K_{\lambda \rho}{ }^{(\mu} \sigma^{\nu) \rho \lambda} .
$$

The independent variables $\theta^{\mu \nu}$ and $\sigma^{\mu \nu \rho}$ are in $1-1$ correspondence with the original variables. The conservation law in the form (3) is the starting point for the derivation of brane world-sheet equations.

In this paper, we are interested in infinitely thin branes, and therefore, restrict our analysis to the singlepole approximation. The multipole expansion of our basic variables then reads:

$$
\begin{gathered}
\theta^{\mu \nu}=\int_{\mathcal{M}} d^{p+1} \xi \sqrt{-\gamma} B^{\mu \nu} \frac{\delta^{(D)}(x-z)}{\sqrt{-g}}, \\
\sigma^{\lambda \mu \nu}=\int_{\mathcal{M}} d^{p+1} \xi \sqrt{-\gamma} C^{\lambda \mu \nu} \frac{\delta^{(D)}(x-z)}{\sqrt{-g}},
\end{gathered}
$$

where $B^{\mu \nu}(\xi)$ and $C^{\lambda \mu \nu}(\xi)$ are the corresponding multipole coefficients. The decomposition (5) is used as an ansatz for solving the conservation equations (3). This has already been done in [13], resulting in manifestly covariant $p$-brane world-sheet equations.

In the next section, the general result of [13] is applied to the $p=2$ case. In particular, the Nambu-Goto type of membrane with totally antisymmetric spin tensor parallel to the world-sheet is thoroughly examined.

\section{MEMBRANE DYNAMICS}

In this section, the stress-energy and spin tensor conservation equations (3) are solved in the single-pole approximation. The general brane world-sheet equations and boundary conditions are applied to the membrane case.

\section{A. Preliminaries}

The $p$-brane world-sheet equations in the single-pole approximation are obtained in the following way. We insert the ansatz (5) into the conservation equations (3), and solve for the unknown variables $z^{\mu}(\xi), B^{\mu \nu}(\xi)$ and $C^{\lambda \mu \nu}(\xi)$. The algorithm for solving this type of equation has been discussed in detail in [12, 13], and here we use the ready-made result. According to [13], the single-pole world-sheet equations are given by

$$
\begin{gathered}
P_{\perp \rho}^{\mu} P_{\perp \sigma}^{\nu} D^{\rho \sigma}=0, \\
\nabla_{a}\left(m^{a b} u_{b}^{\mu}-2 u_{\rho}^{a} D^{\mu \rho}+u_{b}^{\mu} u_{\rho}^{b} u_{\sigma}^{a} D^{\rho \sigma}\right)= \\
=\frac{1}{2} C_{\nu \rho \sigma} \nabla^{\mu} K^{\rho \sigma \nu},
\end{gathered}
$$

while the boundary conditions have the form

$$
\left.n_{a}\left(m^{a b} u_{b}^{\mu}-2 u_{\rho}^{a} D^{\mu \rho}+u_{b}^{\mu} u_{\rho}^{b} u_{\sigma}^{a} D^{\rho \sigma}\right)\right|_{\partial \mathcal{M}}=0 .
$$

Here, $P_{\perp}^{\mu} \equiv \delta_{\nu}^{\mu}-u_{a}^{\mu} u_{\nu}^{a}$ is the orthogonal world-sheet projector, $n^{a}$ is the unit boundary normal, and $\nabla_{a}$ stands for the total covariant derivative that acts on both types of indices:

$$
\nabla_{a} V^{\mu b} \equiv \partial_{a} V^{\mu b}+\left\{\begin{array}{c}
\mu \\
\lambda \rho
\end{array}\right\} u_{a}^{\rho} V^{\lambda b}+\left\{\begin{array}{c}
b \\
c a
\end{array}\right\} V^{\mu c}
$$

The coefficients $m^{a b}(\xi)$ and $C^{\lambda \mu \nu}(\xi)$ are the residual free parameters of the theory. While $m^{a b}$ represents the effective stress-energy tensor of the brane, the $C^{\lambda \mu \nu}$ currents are related to its spin density. The shorthand notation

$$
D^{\mu \nu} \equiv K_{\lambda \rho}^{[\mu} C^{\rho \lambda \nu]}+\frac{1}{2} K_{\lambda \rho}^{[\mu} C^{\nu] \rho \lambda}
$$

is introduced for convenience.

The world-sheet equations (6) and boundary conditions (7) describe the dynamics of an infinitely thin $p$ brane in $D$-dimensional spacetime with curvature and torsion. We want to emphasize that the obtained dynamics has rather universal character. This is because our derivation rests upon the mere existence of the conservation equations of the stress-energy and spin tensors. These conservation equations are the direct consequence of the diffeomorphism and local Lorentz symmetry of the matter Lagrangian. As a consequence of the universality of these symmetries, the conservation equations hold regardless of the details of the theory. This is the virtue of our approach, as it is independent of the particular action used. On the other hand, the conservation equations have the weakness of being incomplete, in the sense that they carry much less information than the full set of field equations. As a consequence, the derived $p$-brane world-sheet equations contain free coefficients. The details of the initial theory are hidden in the form of these coefficients.

By inspecting the form of the obtained world-sheet equations, we realize that only branes made of spinning matter can probe spacetime torsion. Moreover, if the torsion is totally antisymmetric, only axial component of the spin tensor $C^{\lambda \mu \nu}$ survives in the world-sheet equations. In this paper, we shall examine how axial spin tensors couple to torsion. In particular, we are interested in branes characterized by maximally symmetric distribution of stress-energy and spin. It has already been shown in [13] that this task can not be accomplished 
with strings. The reason for this is that the only candidate for the right type of spin-torsion coupling turns out to be the projection of the axial $C^{\lambda \mu \nu}$ on the world-sheet, and it identically vanishes if $p=1$. In what follows, we shall turn to membranes $(p=2)$, and demonstrate how the choice of axial $C^{\lambda \mu \nu}$ parallel to the world-sheet solves the problem.

\section{B. World-sheet equations}

Let us consider an infinitely thin membrane whose spin tensor is totally antisymmetric and parallel to the worldsheet. It is defined by the $C^{\lambda \mu \nu}$ coefficient of the form

$$
C^{\lambda \mu \nu}=s e^{a b c} u_{a}^{\lambda} u_{b}^{\mu} u_{c}^{\nu}
$$

where $e^{a b c}$ is the covariant Levi-Civita symbol, and $s$ is a scalar which measures the spin magnitude. In addition, we shall restrict the scalar $s$ to be a constant, so that our membrane has maximally symmetric distribution of spin. Indeed, the constant $s$ is the only variable in (8) which carries the information about the nature of matter fields the membrane is made of. The rest of $C^{\lambda \mu \nu}$ is given in pure geometric terms. At any given point of the world-sheet, these terms can be gauged away by the proper choice of the target space and world-sheet coordinates. Precisely, we can achieve $g_{\mu \nu}=\eta_{\mu \nu}$ and $u_{a}^{\mu}=\delta_{a}^{\mu}$, so that the non-vanishing components of $C^{\lambda \mu \nu}$ reduce to $C^{a b c}=s \epsilon^{a b c}$. The Levi-Civita symbol $\epsilon^{a b c}$ is a constant tensor of the Lorentz group, which ensures local isotropy of $C^{\lambda \mu \nu}$. As the whole procedure can be repeated with a different choice of the word-sheet point, the $C^{\lambda \mu \nu}$ is a homogeneous tensor, as well. This is exactly what ensures maximal symmetry of the spin tensor.

It is immediately seen that this kind of spin tensor does not exist in the string case. There, the world-sheet is 2-dimensional, and the corresponding Levi-Civita tensor is a second rank tensor. In the membrane case, the world-sheet indices $a, b, \ldots$ take three values, exactly as needed for the existence of the third rank Levi-Civita tensor. As we have seen, this tensor is indispensable for the construction of the maximally symmetric spin tensor.

Let us now see how the membrane spin tensor of the form (8) influences the world-sheet equations (6). First, we calculate the $D^{\mu \nu}$ tensor, and find that it reduces to

$$
D^{\mu \nu}=\frac{3 s}{2} e_{a b c} u^{c[\mu} \stackrel{\mathrm{A}}{K}{ }^{a b \nu]} .
$$

It depends on the axial part of the contorsion

$$
\stackrel{\mathrm{A}}{K}^{\mu \nu \rho} \equiv \frac{1}{3}\left(K^{\mu \nu \rho}+K^{\nu \rho \mu}+K^{\rho \mu \nu}\right)
$$

through its world-sheet projection $\stackrel{\mathrm{A}}{K}{ }^{a b \rho} \equiv u_{\mu}^{a} u_{\nu}^{b} \stackrel{\mathrm{A}}{K}{ }^{\mu \nu \rho}$. The precession equations (6a) are identically satisfied, and we are left with the world-sheet equations $6 \mathrm{~b}$ and boundary conditions (7). Now, we calculate the righthand side of $(6 \mathrm{~b})$, and find

$$
C_{\nu \lambda \rho} \nabla^{\mu} K^{\nu \lambda \rho}=s u_{\nu \lambda \rho} \stackrel{\mathrm{A}}{K^{\mu \nu \lambda \rho}}+\nabla^{c}\left(3 s e_{a b c} \stackrel{\mathrm{A}}{K}^{a b \mu}\right),
$$

with

$$
\stackrel{\mathrm{A}}{K}^{\mu \nu \lambda \rho} \equiv \nabla^{\mu} \stackrel{\mathrm{A}}{K}^{\nu \lambda \rho}-\nabla^{\nu} \stackrel{\mathrm{A}}{K}^{\lambda \rho \mu}+\nabla^{\lambda} \stackrel{\mathrm{A}}{K^{\rho \mu \nu}}-\nabla^{\rho} \stackrel{\mathrm{A}}{K}^{\mu \nu \lambda},
$$

and

$$
u^{\mu \nu \rho} \equiv e^{a b c} u_{a}^{\mu} u_{b}^{\nu} u_{c}^{\rho} .
$$

With the help of (9) and (10), the world-sheet equations are rewritten as

$$
\nabla_{b}\left(\bar{m}^{a b} u_{a}^{\mu}\right)=\frac{s}{2} u_{\nu \lambda \rho} \stackrel{\mathrm{A}}{K}^{\mu \nu \lambda \rho},
$$

where $\bar{m}^{a b} \equiv m^{a b}-\frac{s}{2} \gamma^{a b} K^{\mu \nu \rho} u_{\mu \nu \rho}$ are the residual free coefficients. Using the total antisymmetry of the $\stackrel{\mathrm{A}}{K}^{\mu \nu \lambda \rho}$ field in (11), the coefficients $\bar{m}^{a b}$ are shown to be covariantly conserved,

$$
\nabla_{b} \bar{m}^{a b}=0
$$

This means that Nambu-Goto matter is allowed as the constituent matter of our membrane. Indeed, by demanding

$$
\bar{m}^{a b}=T \gamma^{a b},
$$

where $T$ is a constant commonly interpreted as the membrane tension, the condition (12) is automatically satisfied. At the same time, this choice ensures maximally symmetric distribution of the membrane stress-energy. That it is indeed so can be seen by repeating the arguments used for the demonstration of maximal symmetry of the spin tensor.

Now we are left with only two constants, $T$ and $s$, to characterize our membrane. With this, the world-sheet equations receive their final form

$$
\nabla_{a} u^{a \mu}=\frac{s}{2 T} \stackrel{\mathrm{A}}{K}^{\mu \nu \lambda \rho} u_{\nu \lambda \rho} .
$$

Following the same procedure, the boundary conditions are rewritten as

$$
\left.n^{c}\left(u_{c}^{\mu}+\frac{3 s}{2 T} e_{a b c} \stackrel{\mathrm{A}}{K}^{a b \mu}\right)\right|_{\partial \mathcal{M}}=0 .
$$

The world-sheet equations (14), and the boundary conditions (15) govern the dynamics of a Nambu-Goto type of membrane with axial spin tensor parallel to the worldsheet. Such a membrane is characterized by two constants, the tension $T$ and the spin magnitude $s$, and represents a minimal extension of the Nambu-Goto case.

The equations (14) and (15) are shown to follow from an action functional. Indeed, they are obtained by varying the action

$$
\begin{aligned}
I=T \int d^{3} \xi \sqrt{-h} & {\left[g_{\mu \nu}(x) u_{a}^{\mu} u_{b}^{\nu} h^{a b}+\right.} \\
& \left.+\frac{s}{T} K_{\mu \nu \rho}(x) u_{a}^{\mu} u_{b}^{\nu} u_{c}^{\rho} e^{a b c}-1\right]
\end{aligned}
$$


with respect to the independent variables $x^{\mu}(\xi)$ and $h_{a b}(\xi)$. We cannot help noticing the resemblance of this action to the $\sigma$-model action of [22]. The latter describes an elementary membrane interacting with the 3form field $\mathcal{B}_{\mu \nu \rho}$. In fact, the two actions differ in one instance only: the role of the 3 -form field $\mathcal{B}_{\mu \nu \rho}$ in [22] is played by the contorsion field $\frac{s}{T} \stackrel{\mathrm{A}}{K}_{\mu \nu \rho}$ in (16). Although this matching may be just a coincidence, we find it appropriate to draw the reader's attention to it.

In the next section, we shall demonstrate how the effective string dynamics is obtained in the narrow membrane limit. This way, the dimensionally reduced analogue of the action functional (16) will be obtained.

\section{DIMENSIONAL REDUCTION}

The results of the preceding section are obtained under very general assumptions concerning the dimensionality and topology of spacetime and world-sheet. In what follows, we shall use this freedom to apply these results to a cylindrical membrane wrapped around the extra compact dimension of a $(D+1)$-dimensional spacetime. In the limit of a narrow membrane, we expect to obtain the effective string dynamics. In fact, this kind of double dimensional reduction has already been considered in [22]. There, the string effective action in 10 dimensions has been obtained from the membrane action in 11 dimensions. To complete our exposition, we shall describe a similar $D+1 \rightarrow D$ dimensional reduction.

Let us consider a $(D+1)$-dimensional spacetime with one small compact dimension. It is parametrized by the coordinates $X^{M}(M=0,1, \ldots, D)$, which we divide into the "observable" coordinates $x^{\mu}(\mu=0,1, \ldots, D-1)$, and the extra periodic coordinate $y$. In the limit of small extra dimension, we use the Kaluca-Klein ansatz

$$
\partial_{y} K_{M N L}=0, \quad \partial_{y} G_{M N}=0, \quad G_{y y}=1
$$

to model the contorsion and metric. This ansatz violates the $(D+1)$-dimensional diffeomorphisms, leaving us with the coordinate transformations

$$
x^{\mu^{\prime}}=x^{\mu^{\prime}}(x), \quad y^{\prime}=y+\varepsilon(x) .
$$

In what follows, we shall use the decomposition

$$
G_{M N}=\left(\begin{array}{cc}
g_{\mu \nu}+a_{\mu} a_{\nu} & a_{\mu} \\
a_{\nu} & 1
\end{array}\right)
$$

as it yields the variables that transform as tensors with respect to the residual $D$-diffeomorphisms. The same kind of argument applies to $K_{M N L}$. We shall use a totally antisymmetric contorsion, and decompose it as

$$
K_{L}^{M N} \equiv\left(K_{\lambda}^{\mu \nu}, K_{y}^{\mu \nu}\right) \equiv\left(\mathcal{K}_{\lambda}^{\mu \nu}{ }_{\lambda}+k^{\mu \nu} a_{\lambda}, k^{\mu \nu}\right) .
$$

The components $\mathcal{K}^{\mu \nu}{ }_{\lambda}$ and $k^{\mu \nu}$ are tensors with respect to the residual diffeomorphisms. Now, we consider a membrane wrapped around the extra compact dimension $y$. Its world-sheet $X^{M}=Z^{M}\left(\xi^{A}\right)$ is denoted by $\mathcal{M}_{3}$, and is chosen in the form

$$
x^{\mu}=z^{\mu}\left(\xi^{a}\right), \quad y=\xi^{2},
$$

where the world-sheet coordinates $\xi^{A}(A=0,1,2)$ are divided into $\xi^{a}(a=0,1)$ and $\xi^{2}$. This ansatz reduces the reparametrizations $\xi^{A^{\prime}}=\xi^{A^{\prime}}\left(\xi^{B}\right)$ to

$$
\xi^{a^{\prime}}=\xi^{a^{\prime}}\left(\xi^{b}\right), \quad \xi^{2^{\prime}}=\xi^{2}+\varepsilon\left(z^{\mu}(\xi)\right),
$$

and the world-sheet tangent vectors $U_{A}^{M}=\partial Z^{M} / \partial \xi^{A}$ to

$$
U_{a}^{\mu}=u_{a}^{\mu}, \quad U_{2}^{\mu}=U_{a}^{y}=0, \quad U_{2}^{y}=1 .
$$

One can verify that $u_{a}^{\mu} \equiv \partial z^{\mu} / \partial \xi^{a}$ transforms as a tensor with respect to the residual spacetime and worldsheet diffeomorphisms. The induced metric $\Gamma_{A B} \equiv$ $G_{M N} U_{A}^{M} U_{B}^{N}$ is shown to satisfy the condition $\partial_{2} \Gamma_{A B}=$ 0 . It is decomposed as

$$
\Gamma_{A B}=\left(\begin{array}{cc}
\gamma_{a b}+a_{a} a_{b} & a_{a} \\
a_{b} & 1
\end{array}\right),
$$

with $\gamma_{a b} \equiv g_{\mu \nu} u_{a}^{\mu} u_{b}^{\nu}$, and $a_{a} \equiv a_{\mu} u_{a}^{\mu}$. In what follows, we shall refer to $x^{\mu}=z^{\mu}\left(\xi^{a}\right)$ as the string world-sheet, and denote it by $\mathcal{M}_{2}$.

The membrane boundary $\partial \mathcal{M}_{3}$ is given by $\xi^{A}=$ $\zeta^{A}\left(\lambda^{i}\right)$, where $\lambda^{i}(i=0,1)$ are the boundary coordinates. In accordance with the ansatz (20), it is chosen in the form

$$
\xi^{a}=\zeta^{a}\left(\lambda^{0}\right), \quad \xi^{2}=\lambda^{1} .
$$

The boundary tangent vectors $V_{i}^{A} \equiv \partial \zeta^{A} / \partial \lambda^{i}$ and the boundary normal $N_{A} \equiv e_{A B C} V_{0}^{B} V_{1}^{C}$ are thereby reduced to $v^{a}=\partial \zeta^{a} / \partial \lambda^{0}$ and $n_{a}=e_{a b} v^{b}$, respectively. In what follows, we shall refer to $\xi^{a}=\zeta^{a}\left(\lambda^{0}\right)$ as the string boundary $\partial \mathcal{M}_{2}$.

The membrane world-sheet equations of section III are now rewritten as

$$
\nabla_{A} U^{A M}=\frac{s}{2 T} e_{A B C} U_{N}^{A} U_{L}^{B} U_{R}^{C} K^{M N L R},
$$

with

$$
\begin{aligned}
K^{M N L R} \equiv & \nabla^{M} K^{N L R}-\nabla^{N} K^{L R M}+ \\
& +\nabla^{L} K^{R M N}-\nabla^{R} K^{M N L}
\end{aligned}
$$

Using the Kaluca-Klein ansatz (17), (20), and the decompositions (18), (19), (21) and (22), one finds the following. The $M=y$ component of the world-sheet equations (24) is identically satisfied. The $M=\mu$ components become

$$
\nabla_{a} u^{a \mu}=\frac{3 s}{2 T} e_{a b} u_{\nu}^{a} u_{\lambda}^{b} k^{\mu \nu \lambda}
$$

with

$$
k^{\mu \nu \lambda} \equiv \nabla^{\mu} k^{\nu \lambda}+\nabla^{\nu} k^{\lambda \mu}+\nabla^{\lambda} k^{\mu \nu} .
$$


Similarly, the $M=y$ component of the boundary conditions

$$
\left.N^{C}\left(U_{C}^{M}+\frac{3 s}{2 T} e_{A B C} K^{A B M}\right)\right|_{\partial \mathcal{M}_{3}}=0
$$

is identically satisfied, while $M=\mu$ components reduce to

$$
\left.n^{a}\left(u_{a}^{\mu}+\frac{3 s}{T} e_{a b} k^{\mu b}\right)\right|_{\partial \mathcal{M}_{2}}=0 .
$$

The world-sheet equations (25) and boundary conditions (27) are shown to follow from the action functional

$$
I=T \int d^{2} \xi \sqrt{-h}\left[g_{\mu \nu}(x) h^{a b}+\frac{3 s}{T} k_{\mu \nu}(x) e^{a b}\right] u_{a}^{\mu} u_{b}^{\nu} .
$$

Again, we notice a similarity between our action (28) and the string sigma model action of [16 21]. The latter describes an elementary string coupled to the string axion $\mathcal{B}_{\mu \nu}$. As in the membrane case, the two actions differ in one instance only: the role of the string axion $\mathcal{B}_{\mu \nu}$ in [16 21] is played by the extra dimensional components of the contorsion $\frac{3 s}{T} k_{\mu \nu}$ in (28). Whether this is just a coincidence, or there is more content in this matching is not the subject of our paper. Anyway, it is an interesting observation which, we believe, deserves to be mentioned.

In summary, we have derived how uniform membranes made of spinning matter behave in Riemann-Cartan backgrounds. We have considered a minimal extension of the Nambu-Goto membrane, characterized by two constants only: the tension and spin magnitude. Such membranes proved to be the simplest branes with a nontrivial spin-torsion coupling. The effective string dynamics is obtained from cylindrical membranes in the narrow membrane limit. Both sets of equations are found to follow from corresponding action functionals. By inspecting their form we have discovered a similarity with string theory sigma models.

\section{CONCLUDING REMARKS}

In this paper, we have analyzed the behaviour of classical membranes in Riemann-Cartan backgrounds. The membrane constituent matter is specified in terms of its stress-energy and spin tensors. In particular, the stressenergy is assumed to be of the Nambu-Goto type, and the spin tensor is chosen totally antisymmetric and parallel to the world-sheet. Such membranes have maximally symmetric distribution of stress-energy and spin, and are characterized by two constants only: the tension and spin magnitude. The idea behind these considerations is the search for the simplest brane with nontrivial spin-torsion coupling.

The method we use is a generalization of the Mathisson-Papapetrou method for pointlike matter [1, 2]. It has already been used in [11 13] for the study of strings and higher branes in Riemann-Cartan backgrounds. In this work, the general results of [13] have been applied to a membrane with maximally symmetric distribution of stress-energy and spin. The effective string is seen as a narrow membrane wrapped around the extra spatial dimension.

Our exposition is summarized as follows. In section III we have reviewed the basics of the multipole formalism developed in [11 13]. A manifestly covariant multipole expansion has been defined for an arbitrary exponentially decreasing function, and then applied to the stress-energy and spin tensors of localized matter. The dynamics is specified through the stress-energy and spin tensor covariant conservation equations.

In section III, the conservation equations have been solved for an arbitrary infinitely thin $p$-brane. The resulting manifestly covariant world-sheet equations and boundary conditions have then been applied to the $p=2$ case. The motivation comes from the observation that the needed world-sheet projection of the axial spin tensor vanishes if $p=1$. This kind of spin tensor proved to be the basic ingredient for the construction of a membrane with maximally symmetric distribution of spin. The obtained membrane dynamics has been verified to follow from an action functional. A resemblance with the $\sigma$ model action of [22] has been noted.

In section [V], we have considered cylindrical membranes wrapped around the extra compact dimension of a $(D+1)$-dimensional spacetime. The effective string dynamics is obtained in the narrow membrane limit. It turns out to resemble the string dynamics of $16-21$. Precisely, we have noticed that macroscopic strings couple to torsion the same way as fundamental strings couple to the string axion.

In summary, let us say something about the prospects of our research. We are aware of the fact that considerable additional work can be done along the lines followed in this paper. In fact, our world-sheet equations (6) and boundary conditions (7) contain free parameters that can be chosen in a variety of ways, each leading to a different brane dynamics. The simple choice considered in this paper (membranes with constant tension and spin density), has been shown to lead to the familiar membrane dynamics, and after simple compactification, to the familiar string dynamics. An interesting possibility is to consider more general membranes, and more general compactifications. Hopefully, couplings to the electromagnetic and scalar fields could be discovered in the dimensionally reduced theory. This is, however, a difficult task for itself, and will be considered in a separate paper.

\section{Acknowledgments}

This work is supported by the Serbian Ministry of Science and Technological Development, under Contract No. 141036 . 
[1] M. Mathisson, Acta. Phys. Pol. 6, 163 (1937).

[2] A. Papapetrou, Proc. R. Soc. A 209, 248 (1951).

[3] W. Tulczyjew, Acta. Phys. Pol. 18, 393 (1959).

[4] A. H. Taub, J. Math. Phys. 5, 112 (1964).

[5] G. Dixon, Nuovo Cimento 38, 1616 (1965); 34, 317 (1964); Proc. R. Soc. London 314, 499 (1970); 319, 509 (1970); Gen. Relativ. Gravit. 4, 199 (1973).

[6] A. Trautman, Bull. Acad. Pol. Sci. Ser. Sci. Math. Astron. Phys. 20, 895 (1972).

[7] F. W. Hehl, Phys. Lett. A 36, 225 (1971).

[8] P. B. Yasskin and W. R. Stoeger, Phys. Rev. D 21, 2081 (1980).

[9] K. Nomura, T. Shirafuji, and K. Hayashi, Prog. Theor. Phys. 86, 1239 (1991).

[10] K. Nomura, T. Shirafuji, and K. Hayashi, Prog. Theor. Phys. 87, 1275 (1992).

[11] M. Vasilić and M. Vojinović, Phys. Rev. D 73, 124013 (2006).

[12] M. Vasilić and M. Vojinović, J. High Energy Phys. 07, 028 (2007).
[13] M. Vasilić and M. Vojinović, Phys. Rev. D 78, 104002 (2008).

[14] Y. Nambu, Phys. Rev. D 10, 4262 (1974).

[15] T. Goto, Prog. Theor. Phys. 46, 1560 (1971).

[16] M. B. Green, J. H. Schwarz, and E. Witten, Superstring Theory, Cambridge University Press, Cambridge, 1987.

[17] J. Polchinski, String Theory, Cambridge University Press, Cambridge, 1998.

[18] C. G. Callan, D. Friedan, E. J. Martinec, and M. J. Perry, Nucl. Phys. B262, 593 (1985).

[19] T. Banks, D. Nemeschansky, and A. Sen, Nucl. Phys. B277, 67 (1986).

[20] C. G. Callan, I. R. Klebanov, M. J. Perry, Nucl. Phys. B278, 78 (1986).

[21] E. S. Fradkin and A. A. Tseytlin, Phys. Lett. B 158, 316 (1985); Nucl. Phys. B261, 1 (1985).

[22] M. J. Duff, P. S. Howe, T. Inami and K. S. Stelle, Phys. Lett. B 191, 70 (1987). 